\title{
Bias in estimating food consumption of fish from stomach-content analysis
}

\author{
Rindorf, Anna; Lewy, Peter
}

Published in:

Canadian Journal of Fisheries and Aquatic Sciences

Link to article, DOI:

10.1139/f04-200

Publication date:

2004

Document Version

Peer reviewed version

Link back to DTU Orbit

Citation (APA):

Rindorf, A., \& Lewy, P. (2004). Bias in estimating food consumption of fish from stomach-content analysis. Canadian Journal of Fisheries and Aquatic Sciences, 61(12), 2487-2498. https://doi.org/10.1139/f04-200

\section{General rights}

Copyright and moral rights for the publications made accessible in the public portal are retained by the authors and/or other copyright owners and it is a condition of accessing publications that users recognise and abide by the legal requirements associated with these rights.

- Users may download and print one copy of any publication from the public portal for the purpose of private study or research.

- You may not further distribute the material or use it for any profit-making activity or commercial gain

- You may freely distribute the URL identifying the publication in the public portal

If you believe that this document breaches copyright please contact us providing details, and we will remove access to the work immediately and investigate your claim. 


\title{
Bias in estimating fish consumption by stomach content analysis
}

\author{
A. Rindorf" and P. Lewy \\ Danish Institute for Fisheries Research, Charlottenlund Castle, DK2920 \\ Charlottenlund, Denmark. \\ *Tel: +45 339633 59; Fax: +45 339633 33; email: ar@dfu.min.dk
}


Abstract: This study presents an analysis of the bias introduced by using simplified methods to calculate food intake of fish from stomach contents. Three sources of bias were considered: (1) The effect of estimating consumption based on a limited number of stomach samples, (2) the effect of using average stomach contents derived from

5 pooled stomach samples rather than individual stomachs and (3) the effect of ignoring biological factors which affect the evacuation of prey. Estimating consumption from only two stomach samples provided results close to the actual intake rate in a simulation study. In contrast to this, serious positive bias was introduced by estimating food intake from the contents of pooled stomach samples. An expression is 10 given which can be used to correct analytically for this bias. A new method which takes account of the distribution and evacuation of individual prey types as well as the effect of other food in the stomach on evacuation is suggested for the estimation of the intake of separate prey types. Simplifying the estimation by ignoring these factors biased consumption estimates of individual prey by up to $150 \%$ in a data example.

Keywords: Fish consumption, stomach analyses, food composition, empty stomachs 


\section{Introduction}

The interaction between predatory fish and their prey is a key subject when attempting to understand aquatic ecosystem dynamics. The effect of the predator on the prey relies on the link between diet composition and prey availability and on the total amount of food ingested by the predator. Both diet composition and total consumption are frequently studied by combining knowledge of evacuation rates with field studies of stomach contents. Differing methods have been suggested for this

25 depending on the relationship between stomach content and evacuation rate and on the assumption made on the progress of feeding between consecutive sampling points (Elliot and Persson 1978; Pennington 1985; dos Santos and Jobling 1995). The evacuation of stomach contents of a number of fish species is well described by the relationship

$$
\frac{d S(t)}{d t}=-\rho S^{\alpha}(t)
$$

where $\rho$ is the evacuation rate (in $\left.\mathrm{g}^{1-\alpha} \mathrm{h}^{-1}\right), S$ is stomach content (in $\mathrm{g}$ ) and $\alpha$ is a unitless constant (Jones 1974; Jobling 1981; Andersen 2001). If the predator ingests food at a rate $C(t)$ at time $\mathrm{t}\left(\right.$ in $\mathrm{g} \cdot \mathrm{h}^{-1}$ ), stomach contents vary according to the differential equation 


$$
\frac{d S(t)}{d t}=-\rho S^{\alpha}(t)+C(t)
$$

This equation has been solved analytically for $\alpha=1$ (exponential evacuation) in the case where $C(t)$ is constant over time (Eggers 1977; Elliott and Persson 1978). However, the equation does not have a general analytical solution and in many cases, food intake must be approximated numerically. In the case where $\alpha$ deviates from one and intake rate is not necessarily constant, food intake is usually estimated by methods resembling that suggested by Pennington (1985) who estimated average hourly consumption in a sampling interval as

$$
C_{P}=\frac{\rho}{N} \sum_{i=1}^{N} E\left(S_{i}^{\alpha}\right)+\frac{1}{T}(E(S(T))-E(S(0)))
$$

where $C_{P}$ is average food intake rate in $\mathrm{g}^{-h^{-1}}$ in the sampling interval, $N$ is the number 60 of sampling times in the sampling interval 0 to $T \mathrm{~h}, E(S(0))$ and $E(S(T))$ is the average stomach content (in $\mathrm{g}$ ) of the population sampled at times 0 and $T$, respectively and $E\left(S_{i}^{\alpha}\right)$ is the average of the stomach content to the potency $\alpha$ (in $\mathrm{g}^{\alpha}$ ) 
in the population sampled. $E(S(0))$ and $E(S(T))$ are estimated by the average stomach content of the fish sampled at times 0 and $T$, from hereon denoted $65 \bar{S}(0)$ and $\bar{S}(T)$, whereas $E\left(S_{i}^{\alpha}\right)$ is estimated by $\frac{1}{M_{i}} \sum_{h=1}^{M_{i}} S_{i, h}^{\alpha}$, where $M_{i}$ is the number of stomachs in the $i^{\text {th }}$ sample and $S_{i, h}$ is the contents of the $h^{\text {th }}$ stomach in the $i^{\text {th }}$ sample, from hereon denoted $\overline{S_{i}^{\alpha}}$ (Pennington 1985). Comparable methods have been suggested by a number of authors (Jones 1978; Armstrong 1982; dos Santos and Jobling 1995) while others have used even simpler methods (Daan 1973; Lockwood

70 1980; Basimi and Grove 1985). The degree to which the estimates calculated by any of these methods provide unbiased estimates of food intake rate has hitherto only been investigated for $\alpha=1$ (Eggers 1977; Elliot and Persson 1978; Héroux and Magnan 1996).

The calculation of consumption rate by eq. (2) in cases where $\alpha \neq 1$ requires

75 information on the contents of single stomachs in order to estimate $E\left(S_{i}^{\alpha}\right)$. However, the contents of all stomachs in a sample are often pooled at the time of collection to reduce the cost of examining the composition. $E\left(S_{i}^{\alpha}\right)$ is then estimated as $\left(\bar{S}_{i}\right)^{\alpha}$, where $\bar{S}_{i}$ denotes the observed average stomach content in sample $i$ (Jones 1978; Armstrong 1982; Patterson 1985). As can be shown using Hölder's inequality, this procedure 80 introduces a bias as $E\left(S_{i}^{\alpha}\right)<\left(E\left(S_{i}\right)\right)^{\alpha}$ for $0<\alpha<1$. This bias has been noted by several authors (Ursin et al. 1985; Dos Santos and Jobling 1995; Andersen 2001), and is related to the proportion of stomachs which are classified as empty (Andersen 2001).

Predators with empty stomachs poses an additional problem, as these fish may either be predators caught in the interval between the evacuation of their last meal and 85 the successful capture of the next prey or they may be individuals which are not 
feeding due to other reasons. When the average intake of the total predator population is estimated, the empty stomachs must obviously be included regardless of their origin. However, if only the intake of potentially feeding fish is desired, the individuals which are fasting due to reasons other than the lack of successful capture 90 of prey should be excluded. In the case of spawning fish, this can be done by excluding fish which are ripe or running, but generally the distinction between fasting individuals and feeding fish with empty stomachs is difficult, and this has led to the exclusion of empty stomachs prior to the estimation of food intake (Amundsen and Klemetsen 1988).

95 When the predator feeds on more than one prey species, the amount eaten of each prey is often needed. This has been estimated by distributing the total intake on different prey types according to the weight percentage of the prey in the total stomach content (Armstrong 1982; Hawkins et al. 1985; Persson and Hansson 1999). The implicit assumption is that all prey occur in the average amount in all stomachs and that the evacuation rates of all prey are equal. These assumptions are rarely, if ever, met (Dos Santos and Jobling 1995; Stefánsson and Pálsson 1997; Andersen 1999). Alternatively, the intake of each prey type has been calculated for each prey separately (Armstrong 1982; Patterson 1985). This allows evacuation rate to be varied between prey types. However, the method does not take the effect of other food in the 105 stomach into account. Calculating the intake in this way is therefore based on the assumption that only one prey type is present in each stomach or that evacuation of the prey is independent of other food in the stomach. This may hold in some cases (Magnússon and Aspelund 1997), but in many cases, each stomach contains several prey types (Rice 1988; Albert 1995) and the evacuation of these prey types is not 110 independent of each other (Jones 1974; Andersen 2001). An exception to the 
assumption of separate evacuation is the equation given by Dos Santos and Jobling (1995). However, the parameters in their model have currently only been published for cod (Gadus morhua) and the model furthermore does not allow changes in the average stomach content over time.

This study presents an analysis of biases introduced by making simplifications and erroneous assumptions when estimating food intake. The investigation is divided into three: (1) An examination of the ability of the method suggested by Pennington (1985) to estimate food intake which occurs at a constant rate from two samples, (2) a study of the effect of using average stomach contents 120 derived from pooled stomach samples to estimate food intake and (3) an examination of the effect of ignoring biological factors which affect the evacuation of prey.

The difference between actual food intake and the estimate suggested by Pennington (1985) (number 1 above) is investigated in the case where food intake rate is constant and stomach contents are only sampled twice. The effects of the time 125 elapsed between the sampling points and differences in evacuation rate on bias are examined. Further (number 2 above), an analytical expression for calculating an unbiased estimate of food intake directly from a pooled stomach sample is presented. Lastly (number 3 above), a new estimate of the intake of individual prey types is suggested. The estimate takes the distribution and evacuation of individual prey types 130 into account as well as the effect of other food in the stomach on evacuation rate. The bias introduced by ignoring these factors is analysed and exemplified by estimating food intake of whiting (Merlangius merlangus) of 25 to $30 \mathrm{~cm}$ caught at five different locations in the North Sea. 


\section{Analytical considerations}

\section{Bias of Pennington's method}

The purpose of this exercise was to determine if Pennington's method provides an unbiased estimate of food intake when applied to a predator feeding at a constant rate between two sampling points. This was done by solving equation (1) using numerical integration in a standard mathematical package for a number of

140 combinations of known stomach content at $t=0, S(0)$, food intake rate $C$, time interval $T$ and evacuation rate. The coefficient $\alpha$ was fixed at 0.5 , as this value is appropriate for a number of fish species (Jobling 1981; Andersen 2001). The numerical integration resulted in an estimate of the stomach content at time $T, S(T)$. Using this result, the ability of Pennington's approximation to estimate food intake 145 rate was determined by calculating $C_{P}$ :

$$
C_{P}=\frac{\rho}{2}(\sqrt{S(0)}+\sqrt{S(T)})+\frac{1}{T}(S(T)-S(0))
$$

150

The relative bias was calculated as 


$$
\frac{C_{P}-C}{C}
$$

160

for each combination of $S(0), C, \rho$ and $T$. Relative bias was estimated by for all combinations of $S(0)$ and $C$ in the interval $0.05 \mathrm{~g}$ to $3.05 \mathrm{~g}$. Evacuation rates of 0.04 , 0.14 and 0.24 were used with a fixed time interval of four hours to examine the effect of evacuation rate. These rates correspond to evacuation of slowly evacuated prey in 165 whiting stomachs at $8{ }^{\circ} \mathrm{C}$ and of rapidly evacuated prey at $16^{\circ} \mathrm{C}$. The effect of the length of the interval between consecutive samples was examined by fixing evacuation rate at 0.24 and estimating the difference for $T=1, T=4$ and $T=10$ hours.

\section{Calculating an unbiased estimate of consumption from samples with constant coefficient of variation}

To obtain an estimate of $E\left(S_{i}^{\alpha}\right), \hat{S}_{i}$, directly from the observed average stomach content in a pooled sample, Andersen (2001) proposed a correction factor $\kappa$ which should be multiplied to $\left(\bar{S}_{i}\right)^{\alpha}$ :

175

$$
\hat{S_{i}^{\alpha}}=\kappa\left(\bar{S}_{i}\right)^{\alpha}
$$


180 The correction factor was calculated from a data set consisting of individually analysed saithe (Pollachius virens) stomachs by comparing $\left(\bar{S}_{i}\right)^{\alpha}$ and $\overline{S_{i}^{\alpha}}$. The factor was then applied to a data set consisting mainly of samples where the contents of the non-empty stomachs were pooled prior to weighing. However, if the distribution of the stomach content follows a known distribution, the correction factor can be 185 calculated analytically as well as numerically.

The distribution of the weight of the stomach contents has previously been shown to be reasonably described by delta-distributions based on log-normal- or gamma-distributions (Aitchison and Brown 1957; Stefánsson and Pálsson 1997):

190

$$
\begin{aligned}
& P(S=0)=1-p \\
& P(S \leq s)=p L N\left(s \mid \mu, \sigma^{2}\right), S>0
\end{aligned}
$$

195

or

$$
\begin{aligned}
& P(S=0)=1-p \\
& P(S \leq s)=p \Gamma(s \mid k, \beta) \quad, S>0
\end{aligned}
$$


where $p$ is the probability that a stomach is not empty, $L N\left(s \mid \mu, \sigma^{2}\right)$ and $\Gamma(s \mid k, \beta)$ are 205 the distribution functions of a log-normal distribution with mean $\mu$ and variance $\sigma^{2}$ and a gamma distribution with shape parameter $k$ and scale parameter $\beta$, respectively. In both cases, $E\left(S_{i}^{\alpha}\right)$ can be derived analytically and can be estimated by (Appendix A):

$$
\begin{gathered}
\hat{S_{i}^{\alpha}}=\hat{p}_{i} e^{\frac{1}{2} \alpha \hat{\sigma}_{i}^{2}(\alpha-1)}\left(\bar{x}_{i}\right)^{\alpha} \\
\hat{S_{i}^{\alpha}}=\hat{p}_{i} \frac{\Gamma\left(\hat{k}_{i}+\alpha\right)}{\Gamma\left(\hat{k}_{i}\right) \hat{k}_{i}^{\alpha}}\left(\bar{x}_{i}\right)^{\alpha}
\end{gathered}
$$

in the lognormal and gamma case, respectively, where $\bar{x}_{i}$ denotes the average content of non-empty stomachs in the sample. If the coefficient of variation, $\mathrm{CV}$, of the nonempty stomachs is constant, eqs. (3) and (4) reduces to

$$
\hat{S_{i}^{\alpha}}=\hat{p}_{i} e^{\frac{1}{2} \alpha \hat{\sigma}^{2}(\alpha-1)}\left(\bar{x}_{i}\right)^{\alpha}
$$




$$
\hat{S_{i}^{\alpha}}=\hat{p}_{i} \frac{\Gamma(\hat{k}+\alpha)}{\Gamma(\hat{k}) \hat{k}^{\alpha}}\left(\bar{x}_{i}\right)^{\alpha}
$$

in the lognormal and gamma case, respectively, where $\sigma$ and $k$ are the common parameters for all samples. These parameters must be estimated from stomachs analysed individually, preferably from a sub-sample of the dataset. In the case of a constant $\mathrm{CV}$, equation (5) or (6) can then be used to estimate $E\left(S_{i}^{\alpha}\right)$ for samples where the contents of non-empty stomachs were pooled prior to weighing. $\hat{p}_{i}$ and $\bar{x}_{i}$ can be calculated from these samples whereas $\sigma_{i}$ or $k_{i}$ is unknown. If a sub sample is not available for estimating $\sigma$ or $k$, the parameters could be estimated from a data set consisting of individually analysed stomachs sampled under similar conditions. It is readily apparent that the precision of eqs. (5) and (6) will depend on the degree to 235 which the assumption of constant $\mathrm{CV}$ is fulfilled. This assumption appeared to hold true for the contents of non-empty cod stomachs collected in restricted areas (Stefánsson and Pálsson 1997; Adlerstein and Welleman 2000). However, if it is attempted to estimate the $\mathrm{CV}$ from other data sets, the validity of the assumption should be investigated further.

In addition to the use of eqs. (5) and (6) to correct for pooled samples, they can be applied in cases where stomachs were analysed individually but a common CV could be estimated for the entire dataset. In this case, the simple average $\overline{S_{i}^{\alpha}}$ does not 
provide the best estimate of $E\left(S_{i}^{\alpha}\right)$ and eqs. (5) and (6) provide more precise estimates as the information inherent in a common $\mathrm{CV}$ is taken into account.

245 The estimation of the variance of the estimated consumption has been described by Pennington (1985). However, if eq. (5) or (6) is used to estimate $E\left(S_{i}^{\alpha}\right)$, the equations do not apply. An estimate of the limits of the variance of the consumption is given in Appendix B for both distributions.

Total consumption rate can be estimated by inserting equation (5) or (6) into 250 equation (2). In the case of gamma distributed non-zero stomach contents, $\alpha=0.5$ and a constant $\mathrm{CV}$, the estimation is demonstrated in Table $1\left(C_{1}\right)$. The corresponding estimate derived by simply substituting $\left(\bar{S}_{i}\right)^{\alpha}$ for $E\left(S_{i}^{\alpha}\right)$ is also given $\left(C_{2}\right.$, Table 1$)$. Assuming that $\bar{S}(0)=\bar{S}(T)$, the bias introduced by using $C_{2}$ rather than $C_{1}$ is

$$
\frac{C_{2}-C_{1}}{C_{1}}=\frac{\sum_{i=1}^{N}\left(p_{i} \bar{x}_{i}\right)^{\alpha}}{e^{\frac{1}{2} \alpha \sigma^{2}(\alpha-1)} \sum_{i=1}^{N} p_{i}\left(\bar{x}_{i}\right)^{\alpha}}-1
$$

260 in the case of log-normal distributed contents of non-empty stomachs and 


$$
\frac{C_{2}-C_{1}}{C_{1}}=\frac{\sum_{i=1}^{N}\left(p_{i} \bar{x}_{i}\right)^{\alpha}}{\frac{\Gamma(k+\alpha)}{\Gamma(k) k^{\alpha}} \sum_{i=1}^{N} p_{i}\left(\bar{x}_{i}\right)^{\alpha}}-1
$$

in the case of gamma distributed contents of non-empty stomachs. This bias was estimated for a number of combinations of $p$ and $k$ and $p$ and $\sigma$, respectively, while $\alpha$ was held constant at 0.5 . $p$ was assumed to remain constant over the sampling period.

\section{Estimation of the intake of individual prey types}

Traditional estimates of prey specific intake are based either on the distribution of total intake on prey types according to their weight percentages (Armstrong 1982; Hawkins et al. 1985; Persson and Hansson 1999) or on calculation of the intake of each prey separately. The former method assumes that all prey are

275 present in the average amount in all stomachs and evacuated at the same rate while the assumption of the latter method is that other food in the stomach has no effect on evacuation. Neither of these assumptions are generally met (Dos Santos and Jobling 1995; Stefánsson and Pálsson 1997; Andersen 2001). There is thus a need for an estimate that takes both the effect of the distribution and evacuation of prey individual prey types and the effect of other food in the stomach on evacuation into account.

\section{A new estimate}

The presence of other food in the stomach alters the evacuation of each particular prey (Jones 1974) and the evacuation rate of the total stomach content lies within the range of that of the individual prey types in the stomach (Andersen 2001). 
285 The proportion of all evacuated material which consists of a particular prey is identical to the weight proportion of the prey in the stomach contents (Andersen 2001). In this study, it will be assumed that the evacuation rate of the total content can be approximated by the average of the evacuation rates of the separate prey weighted by the weight of each prey, though this may not be entirely correct (Andersen 2001).

290 The differential equation describing the evacuation of the total stomach content is then:

$$
\frac{d S_{t o t}(t)}{d t}=-\left(\rho_{j} \pi_{j}(t)+\left(1-\pi_{j}(t)\right) \rho_{\text {res }}\right) S_{\text {tot }}^{\alpha}(t)+C_{\text {tot }}(t)
$$

where $\pi_{j}(t)$ denotes the weight proportion of prey species $j$ in the stomach at time $t$ and $S_{\text {tot }}$ denotes the total weight of food in the stomach. The parameters all refer to stomachs where prey $j$ is present as the prey obviously does not affect evacuation in stomachs where it is absent. $\rho_{\mathrm{j}}$ is the evacuation rate of the prey in question and $\rho_{\text {res }}$ is the evacuation rate of the other stomach content (excluding prey $j$ ). The differential equation describing the evacuation of prey $j$ becomes 


$$
\frac{d S_{j}(t)}{d t}=-\pi_{j}(t)\left(\rho_{j} \pi_{j}(t)+\left(1-\pi_{j}(t)\right) \rho_{\text {res }}\right) S_{\text {tot }}^{\alpha}(t)+C_{j}(t)
$$

310 Recall that the proportion of the total amount of food evacuated which consists of prey $j$ is identical to the weight proportion of $j$ in the stomach where the prey occurs, i.e.,

$$
\pi_{j}(t)=\frac{S_{j}(t)}{S_{\text {tot }}(t)}
$$

eq. (10) can also be written as

$$
\frac{d S_{j}(t)}{d t}=-\pi_{j}^{1-\alpha}(t)\left(\pi_{j}(t) \rho_{j}+\left(1-\pi_{j}(t)\right) \rho_{\text {res }}\right) S_{j}^{\alpha}(t)+C_{j}(t)
$$

$\bar{C}_{j}$ can then be estimated by 


$$
C_{3, j}=\frac{1}{N} \sum_{i=1}^{N} \pi_{j, i}^{1-\alpha}\left(\pi_{j, i} \rho_{j}+\left(1-\pi_{j, i}\right) \rho_{r e s}\right) E\left(S_{j, i}^{\alpha}\right)+\frac{1}{T}\left(E\left(S_{j}(T)\right)-E\left(S_{j}(0)\right)\right)
$$

(Table 1). The subscript 3 distinguishes this estimate from those obtained by other 335 methods. This new estimate thus takes both the uneven distribution of prey in the stomachs and the effect of other stomach content on the evacuation of the particular

prey into account. $E\left(S_{i}^{\alpha}\right)$ can be estimated directly from individually analysed stomachs or by equation (5) or (6). In the latter case, an estimate of the occurrence of the prey, $p_{j}$, is required as well as an estimate of $\sigma_{j}$ or $k_{j}$.

\section{Comparison between the new estimate and traditional methods}

A number of authors estimate the intake of individual prey types by distributing the total intake according to the weight percentages of the different prey (Armstrong 1982; Hawkins et al. 1985; Seyhan and Grove 1998);

$$
C_{4, j}=\pi_{\text {tot }, j} C_{1}
$$


where $\pi_{\mathrm{tot}, j}$ is the weight percentage of $j$ in the contents of all stomachs and $C_{1}$ is the total food intake. As shown in Appendix C, this estimate is biased even in the case where both evacuation rates and $\mathrm{CVs}$ of the weight of the stomach contents are identical for prey $j$ and the total stomach contents. The effect on the bias of the proportion of stomachs which contain the particular prey, $p_{j}$, relative to the probability that a stomach contains food of any kind, $p_{t o t}$, and the effect of the ratio between the

355 weight proportion of the prey in all stomachs, $\pi_{t o t, j}$ and in stomachs where the prey occurred, $\pi$, was investigated in the special case where $\rho_{j}=\rho_{\text {tot }}, k_{j}=k_{\text {tot }}$, no change occurred in the average stomach content over the sampling period and $\pi_{\text {tot, } j}$ and $\pi_{j}$ remain constant over the period. In this case, the relative bias is reduced to

$$
\frac{C_{4}-C_{3}}{C_{3}}=\left(\frac{\pi_{t o t, j}}{\pi_{j}} \frac{p_{t o t}}{p_{j}}\right)^{1-\alpha}-1
$$

(Appendix C). This bias was plotted as a function of the ratios $\frac{\pi_{t o t, j}}{\pi_{j}}$ and $\frac{p_{t o t}}{p_{j}}$ for $\alpha=0.5$. The plot illustrates the bias introduced even in the case where evacuation rate does not vary between prey types.

An alternative procedure is to calculate the intake of each prey type separately 370 (Armstrong 1982; Patterson 1985). The assumption is that other food in the stomach 
has no effect on evacuation. The differential equation describing the stomach content then becomes

$$
\frac{d S_{j}(t)}{d t}=-\rho_{j} S_{j}^{\alpha}(t)+C_{j}(t)
$$

and food intake rate can be calculated as

$$
C_{5 j}=\frac{\rho_{j}}{N} \sum_{i=1}^{N} E\left(S_{j, i}^{\alpha}\right)+\frac{1}{T}\left(E\left(S_{j}(T)\right)-E\left(S_{j}(0)\right)\right)
$$

The formula is thus identical to eq. (2), except that it refers only to the weight and evacuation rate of prey $j$. If $\pi_{j}$ remains constant over the period, the bias introduced by this method reduces to 


$$
\frac{C_{5, j}-C_{3, j}}{C_{3, j}}=\frac{\rho_{j}}{\left(\pi_{j} \rho_{j}+\left(1-\pi_{j}\right) \rho_{\text {res }}\right) \pi_{j}^{1-\alpha}}-1
$$

(Appendix C). This bias was plotted as a function of $\rho_{\text {res }} / \rho_{\mathrm{j}}$ and $\pi_{\mathrm{j}}$.

\section{Materials and methods}

\section{Data set}

The data consisted of whiting of a length of $25-30 \mathrm{~cm}$ gathered around the clock at five locations in the North Sea. Trawl hauls were performed with four hour intervals for a total of 48-72 hours. The sampling procedure has been described in detail elsewhere (Rindorf 2002) as has the calculation of the occurrence and weight of different prey types (Rindorf 2003, 2004). The total weight present in non-empty stomachs and the occurrence of non-empty stomachs were analysed by models similar to the analyses of total weight and occurrence of the single prey described in Rindorf (2003, 2004). Briefly, the effect of time of day, time elapsed since the first sample was taken and location on occurrence and mean weight of single prey and total stomach content was analysed. If a factor was found to have no significant effect on

410 weight or occurrence, samples were joined prior to estimation of mean weight or occurrence, respectively. This procedure was used to minimize random errors in the estimates. The weight of both single prey and total stomach content in stomachs where this exceeded zero was assumed to be gamma distributed with a constant CV. 
The average hourly food intake was calculated from the estimated average

415 stomach content in non-empty stomachs, the estimated common shape parameter, $k$, and the occurrence of non-empty stomachs, $p$. The estimation of these parameters was described in detail in Rindorf $(2003,2004)$.

The bias introduced by not correcting for the inequality between $\left(\bar{S}_{i}\right)^{\alpha}$ and $\overline{S_{i}^{\alpha}}$ was examined by estimating the total consumption as $C_{1}$ (correcting) and $C_{2}$ (not 420 correcting)(Table 1). The bias introduced by the two simplified methods for calculating intake of individual prey was investigated by estimating the intake of three different prey types by $C_{3}, C_{4}$ and $C_{5}$ (Table 1). Benthic invertebrates were chosen as an example of a prey that was generally small, occurred frequently in the stomachs and had a high evacuation rate. Herring (Clupea harengus) was chosen as an

425 infrequent large prey with an evacuation rate close to that of the total stomach content. Finally, crabs were selected to exemplify prey that occurred frequently and posses a hard exoskeleton and thus are evacuated at a low rate.

\section{Estimation of evacuation rates, $\pi_{t o t, j}$ and $\pi_{j}$}

Evacuation rate is dependent on both temperature and predator and prey characteristics. Additionally, evacuation rate has been found to vary with meal size in some cases (Garber 1983; Elliot 1991; Temming and Andersen 1994), but as this effect has been shown to be insignificant in other cases (Bromley 1987; Andersen 1998), it was not considered in this study. The estimation of evacuation rates is described in detail in Appendix D and the resulting values are given in Table 2. The

435 percentage of the total stomach contents which consisted of each prey, $\pi_{\text {tot }, j}$, was calculated at each location and time of day. In addition, the percentage of the stomach content which was made up by prey $j$ in the stomachs where prey $j$ occurred, $\pi$, was 
calculated for each prey, location and time of day. $\pi_{j}$ was thus assumed to be constant and statistically independent of $S_{j}$ for a given time of day and location. As changes in

$440 S_{j}$ occurred through changes in $p_{j}$ rather than in $\bar{x}_{j}$ (though trends in the former occurred over the sampling period, no trends were detected in the latter (Rindorf 2003, 2004)), this assumption appeared justified.

\section{Result}

\section{Bias of Pennington's method}

445 The bias was small for short intervals and in no case exceeded $3 \%$ when the time interval was one hour. However, increasing the interval led to large differences when the stomach content or intake was low (Fig. 1). The bias rose to $+34 \%$ at a 10 hour sampling interval and a very low food intake rate. The difference between the two estimates increased with evacuation rate, being virtually zero for an evacuation rate of 0.04 (maximum bias $-1.4 \%$ ) rising to up to a maximum of $8 \%$ during a four hour interval at an evacuation rate of 0.24 (Fig. 1). For the large majority of combinations of $C$ and $S(0)$, the difference was less than $5 \%$ if the sampling interval was four hours or less.

\section{Calculating an unbiased estimate of consumption from samples with constant} coefficient of variation

Substantial bias was introduced by estimating $E\left(\sqrt{S_{i}}\right)$ as $\sqrt{\bar{S}_{i}}$. The bias increased dramatically as a function of $\sigma^{2}$ and $k^{-1}$ in the log-normal and gamma distributed data, respectively (Fig. 2). Empty stomachs further increased the bias, leading to a maximum of several hundred per cent in both distributions. The 460 consumption calculated from the whiting data was increased by $51 \%$, on average, 
when $E\left(\sqrt{S_{i}}\right)$ was estimated by $\sqrt{\bar{S}_{i}}$ (Table 3$)$. The common shape parameter $k$ of the distribution of the total stomach contents was 0.269 and the proportion of stomachs which contained food was around 0.8 (Table 3).

\section{Estimation of prey specific intake}

465 Distributing the total intake on prey types according to weight percentage seriously biased the estimates (Fig. 3). The area in which the bias was less than $20 \%$ constituted a very small part of the possible parameter combinations. If the prey was found in most of the non-empty stomachs, the bias was generally negative. As the occurrence of the prey in the non-empty stomachs decreased, positive bias became

470 more likely. The data example demonstrated this as the estimate $C_{4, j}$ was lower than $C_{3, j}$ when the prey occurred frequently in the stomachs (Table 4). The estimate $C_{4, j}$ exceeded $C_{3, j}$ at low occurrences, in particular where the prey constituted less than three quarters of the stomach contents in the stomachs where it occurred. $C_{4, j}$ was biased by $-36 \%$ and $-44 \%$ on average for benthic invertebrates and crabs, respectively, 475 when intake exceeded $3 \mathrm{mg} \cdot \mathrm{h}^{-1}$. Herring estimated by $C_{4, j}$ was positively biased at location 3 though the occurrence was similar to that at location 1 where a slight negative bias was found. This was caused by a higher mean weight of herring at location 3 (Appendix C).

Failing to include the effect on evacuation of other food in the stomach on

480 introduced a positive bias on consumption. When the prey in question constituted half the stomach content weight, consumption was estimated to be approximately $140 \%$ of the true consumption even when evacuation rate of the prey was identical to that of the total stomach content (Fig. 4). However, as the prey often constituted the majority of the stomach content when present in the whiting stomachs, the positive bias on 
estimated consumption was only $26 \%, 9 \%$ and $10 \%$ on average for benthic invertebrates, herring and crabs, respectively (Table 4). The relative size of bias of the three groups reflected that expected by the size of the evacuation rate of the prey and the proportion of the stomach content made up by this prey (Table 4).

\section{Discussion}

The bias introduced by using Pennington's method to estimate a constant food intake rate was very small for sampling intervals of four hours or less. In contrast to this, simplifying calculation of input data and making unwarranted assumptions regarding the distribution of prey in the stomachs led to serious bias in the estimated food intake.

The estimation of $E\left(S_{i}^{\alpha}\right)$ by $\left(\bar{S}_{i}\right)^{\alpha}$ led to substantial overestimation of consumption. This effect was also noted by Ursin et al. (1985), Dos Santos and Jobling (1995) and Andersen (2001) using different methods. The latter author estimated correction factors that could be applied to eliminate bias from data consisting of individually analysed stomachs. Though individual stomachs are also needed to estimate the parameters in the correction suggested here, the two methods differ in their underlying assumptions. Whereas Andersen's (2001) correction factor assumes a constant coefficient of variation of the contents of non-empty stomachs as well as a constant proportion of empty stomachs, the present method allows these assumptions to be made independently of each other, ensuring a minimum number of 505 fixed parameters. Further, the method presented here provides a lower limit of the variance of the estimated consumption. The distribution and coefficient of variation can be estimated from a sub sample of the dataset, and the remaining data can then be analysed by counting the number of empty and non-empty stomachs and recording the 
mean weight of the contents of the non-empty stomachs. This sampling procedure has traditionally been followed in a number of stomach sampling projects (ICES 1988; 1991). The correction requires a constant $\mathrm{CV}$, an assumption that may not hold if extensive areas or time scales are considered. There does, however, appear to be some evidence of constancy, as Stefánsson and Pálsson (1997) and Adlerstein and Welleman (2000) found evidence of a common CV of the contents of non-empty 515 stomachs of Icelandic and North Sea cod, respectively. Nevertheless, the assumption is of crucial importance to the validity of the correction, and should be considered in detail before attempting to correct large numbers of samples.

Simply excluding empty stomachs leads to an overestimate of food intake as the fish which were caught in the interval between the complete evacuation of their 520 last meal and the time of their successful capture of the next prey are excluded. Empty stomachs should only be excluded when there is biological evidence to suggest that these fish were indeed not part of the feeding population. In this study, there was no evidence to suggest that some predators may have been fasting. However, if some predators appeared to have decreased their food intake due to e.g. spawning, the

525 biologically most appropriate estimate of food intake would appear to be separate estimates for spawning and non-spawning fish, hence considering empty and nonempty stomachs of spawning fish together.

The new method for calculating the consumption of individual prey provided greatly improved estimates. Compared to this method, consumption of the three prey 530 types considered was seriously biased by distributing the total consumption on prey types using the weight percentages. Though the bias was generally negative, positive bias was found for prey, which occurred infrequently or constituted a minor part of the stomach content when present. In general, larger fish prey occur only in a minor 
percentage of the stomachs collected (Albert 1995; Pillar and Barange 1997) and so consumption of fish is likely to be overestimated whereas that of invertebrates will be underestimated. This conclusion holds even when no difference in evacuation rate exists between the two prey types. Fish in which the evacuation of the stomach content is exponential $(\alpha=1)$ represents a special case in which the error in distributing the total consumption according to weight percentages relies solely on the difference in evacuation rates between prey (Appendix C).

The effect of other prey on evacuation and hence consumption was minor in most cases. Only the intake of benthic invertebrates was biased to any extent by this assumption and if simplifications of the calculations are to be made, the assumption of evacuation of each prey separately was far superior to distributing consumption

545 according to weight percentages. Unfortunately, studies calculating the intake of single prey by this method frequently estimate $E\left(S_{i}^{\alpha}\right)$ as $\left(\overline{S_{i}}\right)^{\alpha}$ (Armstrong 1982; Patterson 1985) and their results are therefore positively biased.

Estimating the intake of each prey type in the data set and summing these to obtain the total intake led to estimates of total intake of $62-131 \%$ of $C_{1}$ in the data 550 analysed here. This difference may be the result of a combination of random errors and a tendency to overestimate the mean weight of highly digested prey, as prey is not identified during the last stages of digestion. However, the difference may also reflect a biologically more sensible method of calculation. The calculation of an average evacuation rate of the total stomach content fails to account for any correlation 555 between evacuation rate and weight of the stomach content. Such correlation may arise if the stomachs contain e.g. either small crustaceans (low evacuation rate) or large lean fish (high evacuation rate). 
In addition to the effect of using different methods to estimate consumption, the present study demonstrated the importance of collecting samples throughout the diel cycle: The average intake of crabs estimated from daytime samples alone was only $25-50 \%$ of that estimated by night time samples in the data at hand. This was connected to the primarily nocturnal feeding on crabs (Rindorf 2003). Sampling during daytime only would therefore lead to gross underestimation of the total intake of this prey type.

The new method for calculating the intake of individual prey has the advantage of incorporating the known sources of bias in food intake estimation while allowing average stomach content to change during the sampling period. A major disadvantage is, however, the expense of collecting detailed information on the contents of individual stomachs. If the coefficient of variation is constant for each prey, the need for information could be limited to the average weight, the $\mathrm{CV}$ of this and the occurrence of each prey type. The analyses of the stomachs would then not require the weighing of prey from each stomach individually, except in the sub sample necessary to estimate the correction factor. The effect of other food in the stomach on estimated consumption appears to be minor in most cases.

575 In conclusion, the estimation of $E\left(S_{i}^{\alpha}\right)$ as $\left(\bar{S}_{i}\right)^{\alpha}$ seriously biases the calculation of food intake by fish. The bias increases with the variance of the contents of the nonempty stomachs and rises dramatically if the proportion of empty stomachs is high. If single stomach data or coefficients of variation are not available, $E\left(S_{i}^{\alpha}\right)$ should therefore at the very least be estimated as $p_{i}\left(\bar{X}_{i}\right)^{\alpha}$, as this would eliminate the severe effect of empty stomachs. Distributing the total food intake on different prey types according to their weight percentage produces large biases, which may be either positive or negative according to the distribution and evacuation rate of the prey. As 
this bias can take any value, it is even more dangerous than the bias of the simplified calculations, as the latter is at least known to be positive. The results shown here once

585 again stress the need for information on stomach contents on a single stomach level. If this type of information cannot be obtained, great care should be taken to minimize the otherwise substantial bias.

\section{Acknowledgements}

We would like to thank two anonymous referees whose comments helped us

590 to improve the manuscript. This study was partly funded by a grant from the Danish Ministry of Food, Agriculture and Fisheries to the project 'Development of improved models of fisheries impact on marine fish stocks and ecosystems' (FIFOF-DFU-1) and by the EU project 'Development of structurally detailed statistically testable models of marine populations' (QLK5-CT1999-01609). The research was supported 595 by the SLIP research school under the Danish Network for Fisheries and Aquaculture Research (www.fishnet.dk) financed by the Danish Ministry for Food, Agriculture and Fisheries and the Danish Agricultural and Veterinary Research Council. 


\section{References}

600 Adlerstein, S. A., and Welleman, H. C. 2000. Diel variation of stomach contents of North Sea cod (Gadus morhua) during a 24-h fishing survey: an analysis using generalized additive models. Can. J. Fish. Aquat. Sci. 57: 2363-2367.

Aitchison, J., and Brown, J. A. C. 1957. The lognormal distribution with special reference to its use in economics. Cambridge University Press, Cambridge.

605 Albert, O. T. 1995. Diel changes in food and feeding of small gadoids on a costal bank. ICES J. Mar. Sci. 52: 873-885.

Amundsen, P. A., and Klemetsen, A. 1988. Diet, gastric evacuation rates and food consumption in a stunted population of Arctic charr, Salvelinus alpinus L., in Takvatn, northern Norway. J. Fish Biol. 33: 697-709.

610 Andersen, N. G. 1998. The effect of meal size on gastric evacuation in whiting. J. Fish Biol. 52: 743-755.

Andersen, N. G. 1999. The effects of predator size, temperature, and prey characteristics on gastric evacuation in whiting. J. Fish Biol. 54: 287-301.

Andersen, N. G. 2001. A gastric evacuation model for three predatory gadoids and 615 implications of using pooled field data of stomach contents to estimate food rations. J. Fish Biol. 59: 1198-1217.

Armstrong, M. J. 1982. The predator-prey relationships of Irish Sea poor-cod (Trisopterus minutus L.), pouting (Trisopterus luscus L.), and cod (Gadus morhua L.). J. Cons. Int. Explor. Mer 40: 135-152. 
620 Basimi, R. A., and Grove, D. J. 1985. Estimates of daily food intake by an inshore population of Pleuronectes platessa L. off eastern Anglesey, North Wales. J. Fish Biol. 27: 505-520.

Bromley, P. J. 1987. The effects of food type, meal size and body weight on digestion and gastric evacuation in turbot, Scophthalmus maximus L. J. Fish Biol., 30: $501-512$.

Daan, N. 1973. A quantitative analyses of the food intake of North Sea cod, Gadus morhua. Neth. J. Sea Res. 6: 479-517.

Dos Santos, J., and Jobling, M. 1995. Test of a food consumption model for the Atlantic cod. ICES J. Mar. Sci. 52: 209-220.

630 Eggers, D. M. 1977. Factors in interpreting data obtained by diel sampling of fish stomachs. J. Fish. Res. Board Can. 34: 290-294.

Elliott, J. M. 1991. Rates of gastric evacuation in piscivorous brown trout, Salmo trutta. Freshwat. Biol. 25: 297-30.

Elliott, J. M., and Persson, L. 1978. The estimation of daily rates of food consumption 635 for fish. J. Anim. Ecol. 47: 977-991.

Garber, K. J. 1983. Effect of fish size, meal size and dietary moisture on gastric evacuation of pelleted diets by yellow perch, Perca flavescens. Aquaculture 34: 41-49.

Hawkins, A. D., Soofiani, N. M., and Smith, G. W. 1985. Growth and feeding of 640 juvenile cod, Gadus morhua (L). J. Cons. Int. Explor. Mer 42: 11-32.

Héroux, D., and Magnan, P. 1996. In situ determination of food daily ration in fish: review and field evaluation. Environ. Biol. Fish. 46: 61-74. 
ICES 1988. Report of the meeting of the coordinators in the stomach sampling project 1985 to 1987. ICES (Int. Counc. Explor. Sea) C.M. 1988/G:27. Available

645 from the International Council for the Exploration of the Sea, Palaegade 2-4, 1261 K, Copenhagen, Denmark.

ICES 1991. Manual for the ICES North Sea stomach sampling project in 1991. ICES (Int. Counc. Explor. Sea) C.M. 1991/G:3. Available from the International Council for the Exploration of the Sea, Palaegade 2-4, $1261 \mathrm{~K}$, Copenhagen, Denmark.

Jobling, M. 1981. Mathematical models of gastric emptying and the estimation of daily rates of food consumption by fish. J. Fish Biol. 19: 245-257.

Jones, R. 1974. The rate of elimination of food from the stomachs of haddock Melanogrammus aeglefinus, cod Gadus morhua, and whiting Merlangius merlangus. J. Cons. Int. Explor. Mer 35: 225-243.

Jones, R. 1978. Estimates of food consumption of haddock (Melanogrammus aeglefinus) and cod (Gadus morhua). J. Cons. Int. Explor. Mer 15: 225-243.

Lewy, P. 1996. A generalized dirichlet distribution accounting for singularities of the variables. Biometrics 52: 1394-1409.

660 Lockwood, S. J. 1980. The daily food intake of 0-group plaice (Pleuronectes platessa L.) under natural conditions. J. Cons. Int. Explor. Mer 39: 154-159.

Magnússon, K. G., and Aspelund, T. 1997. A model for estimating meal frequency and meal size from stomach data with an application to Atlantic cod (Gadus morhua) feeding on capelin (Mallotus villosus). Can. J. Fish. Aquat. Sci. 54: 876-889. 
Patterson, K. R. 1985. The trophic ecology of the whiting (Merlangius merlangus) in the Irish Sea and its significance to the Manx herring stock. J. Conc. Int. Explor. Mer 42: 152-161.

Pedersen, J., and Hislop, J. R. G. 2001. Seasonal variations in the energy density of 670 fishes in the North Sea. J. Fish Biol. 59: 380-389.

Pennington, M. 1985. Estimating the average food consumption by fish in the field from stomach contents data. Dana 5: 81-86.

Persson, A., and Hansson, L.-A. 1999. Diet shift in fish following competitive release. Can. J. Fish. Aquat. Sci. 56: 70-78.

675 Pillar, S. C., and Barange, M. 1997. Diel variability in bottom trawl catches and feeding activity of the Cape hakes off the west coast of South Africa. ICES J. Mar. Sci. 54: 485-499.

Rice, J. C. 1988. Repeated cluster analysis of stomach contents data: method and application to diet of cod in NAFO division 3L. Env. Biol. Fish. 21: 263-277.

680 Rindorf, A. 2002. The effect of stomach fullness on food intake of whiting in the North Sea. J. Fish Biol. 61: 579-593.

Rindorf, A. 2003. Diel feeding pattern and prey choice of whiting (Merlangius merlangus) in the North Sea. Mar. Ecol. Prog. Ser. 249: 265-276.

Rindorf, A. 2004. Estimation of evacuation rates in the field. In press, J. Fish Biol.

685 Seyhan, K., and Grove, D. J. 1998. Food consumption of whiting, Merlangius merlangus, in the Eastern Irish Sea. Fish. Res. 38: 233-245.

Stefánsson, G., and Pálsson, O. K. 1997. Statistical evaluation and modelling of the stomach contents of Icelandic cod (Gadus morhua). Can. J. Fish. Aquat. Sci. 54: $169-181$. 
690 Temming, A., and Andersen, N. G. 1994. Modelling gastric evacuation without meal size as a variable. A model applicable for the estimation of daily ration of cod (Gadus morhua L.) in the field. ICES J. Mar. Sci. 51: 429-438.

Ursin, E., Pennington, M., Cohen, E. B., and Grosslein, M. D. 1985. Stomach evacuation rates of Atlantic cod (Gadus morhua) estimated from stomach 695 contents and growth rates. Dana 5: 63-80. 


\section{Appendix A}

Estimation of $E\left(S_{i}^{\alpha}\right)$ from $E\left(S_{i}\right)$

Let the distribution of the weight of the stomach contents, $S$, be described by a

700 delta-distribution based on log-normal- or gamma-distributions (Aitchison and Brown 1957; Stefánsson and Pálsson 1997):

$$
\begin{aligned}
& P(S=0)=1-p \\
& P(S \leq s)=p L N\left(s \mid \mu, \sigma^{2}\right), S>0
\end{aligned}
$$

or

$$
\begin{aligned}
& P(S=0)=1-p \\
& P(S \leq s)=p \Gamma(s \mid k, \beta) \quad, S>0
\end{aligned}
$$

where $p$ is the probability that a stomach is not empty, $L N\left(s \mid \mu, \sigma^{2}\right)$ and $\Gamma(s \mid k, \beta)$ are the distribution functions of a log-normal distribution with mean $\mu$ and variance $\sigma^{2}$ and a gamma distribution with shape parameter $k$ and scale parameter $\beta$, respectively. 
In both cases, $E\left(S_{i}^{\alpha}\right)$ can be derived analytically. Let the weight of the contents of a non-empty stomach be described by $X$, where $X$ is log-normal distributed with mean $\mu$ and variance $\sigma^{2}$ of the $\log$ stomach contents:

$$
X \in L N\left(\mu, \sigma^{2}\right)
$$

then

$$
X^{\alpha} \in L N\left(\alpha \mu, \alpha^{2} \sigma^{2}\right)
$$

The relation between $(E(X))^{\alpha}$ and $E\left(X^{\alpha}\right)$ is

$$
E\left(X^{\alpha}\right)=e^{\frac{1}{2} \alpha \sigma^{2}(\alpha-1)}(E(X))^{\alpha}
$$


745 and $E\left(S^{\alpha}\right)$ becomes

$$
E\left(S^{\alpha}\right)=p e^{\frac{1}{2} \alpha \sigma^{2}(\alpha-1)}(E(X))^{\alpha}
$$

In the case of gamma distribution, $X \in \Gamma(k, \beta), E\left(X^{\alpha}\right)$ is

755

$$
E\left(X^{\alpha}\right)=\frac{\Gamma(k+\alpha)}{\Gamma(k) k^{\alpha}}(E(X))^{\alpha}
$$

and

760

$$
E\left(S^{\alpha}\right)=p \frac{\Gamma(k+\alpha)}{\Gamma(k) k^{\alpha}}(E(X))^{\alpha}
$$


Hence $E\left(S_{i}^{\alpha}\right)$ can be estimated by

$$
\begin{aligned}
& \hat{S_{i}^{\alpha}}=\hat{p}_{i} e^{\frac{1}{2} \alpha \hat{\sigma}_{i}^{2}(\alpha-1)}\left(\bar{x}_{i}\right)^{\alpha} \\
& \hat{S_{i}^{\alpha}}=\hat{p}_{i} \frac{\Gamma\left(\hat{k}_{i}+\alpha\right)}{\Gamma\left(\hat{k}_{i}\right) \hat{k}_{i}^{\alpha}}\left(\bar{x}_{i}\right)^{\alpha}
\end{aligned}
$$

775 in the lognormal and gamma case, respectively. 


\section{Appendix B}

\section{Estimation of the variance of the estimated consumption}

The variance of the estimated consumption can be estimated by

780

$$
V(\hat{C})=\frac{\rho^{2}}{N^{2}} \sum_{i=1}^{N} V\left(\hat{S_{i}^{\alpha}}\right)+\frac{1}{T^{2}}(V(\hat{S(T)})+V(\hat{S(0)}))
$$

785

in the case where $S(0)$ and $S(T)$ are not included in the summation and

790

$V(\hat{C}) \cong \frac{\rho^{2}}{N^{2}} \sum_{i=1}^{N} V\left(\hat{S_{i}^{\alpha}}\right)+\frac{1}{T^{2}}(V(\hat{S(T)})+V(\hat{S(0)}))+\frac{\alpha \rho}{N T}\left(\left(S(\hat{T)})^{\alpha-1} V(\hat{S(T)})-(\hat{S(0)})^{\alpha-1} V(\hat{S(0)})\right)\right.$

795 in the case where $S(0)$ and $S(T)$ are included in the summation. To calculate this variance, an estimate of the variance of both $\hat{S(0)}, \hat{S(T)}$ and $\hat{S_{i}^{\alpha}}$ are needed. If it is assumed that $\sigma$ and $k$ are constant and known without error, the variance of $\hat{S}_{i}^{\alpha}$ can be approximated by 
800

$$
V\left(\hat{S}_{i}^{\alpha}\right) \cong e^{\alpha \sigma^{2}(\alpha-1)}\left(V\left(\hat{p}_{i}\right)\left(\bar{X}_{i}\right)^{2 \alpha}+\alpha\left(\bar{X}_{i}\right)^{\alpha-1} V\left(\bar{X}_{i}\right) \hat{p}_{i}{ }^{2}+\alpha\left(\bar{X}_{i}\right)^{\alpha-1} V\left(\bar{X}_{i}\right) V\left(\hat{p}_{i}\right)\right)
$$

805

and

$$
V\left(\hat{S_{i}^{\alpha}}\right) \cong\left(\frac{\Gamma(k+\alpha)}{\Gamma(k) k^{\alpha}}\right)^{2}\left(V\left(\hat{p}_{i}\right)\left(\bar{X}_{i}\right)^{2 \alpha}+\alpha\left(\bar{X}_{i}\right)^{\alpha-1} V\left(\bar{X}_{i}\right) \hat{p}_{i}{ }^{2}+\alpha\left(\bar{X}_{i}\right)^{\alpha-1} V\left(\bar{X}_{i}\right) V\left(\hat{p}_{i}\right)\right)
$$

in the lognormal and gamma distributed case, respectively. 


$$
V\left(\hat{S_{i}^{\alpha}}\right) \cong \frac{V\left(S_{i}^{\alpha}\right)}{M_{i}}=\frac{1}{M_{i}} \hat{p}_{i}\left(e^{2 \hat{\mu}_{i}+\hat{\sigma}_{i}^{2}}\right)^{\alpha}\left(e^{\alpha \hat{\sigma}_{i}^{2}(2 \alpha-1)}-\hat{p}_{i} e^{\alpha \hat{\sigma}_{i}^{2}(\alpha-1)}\right)
$$

825 and

$$
V\left(\hat{S_{i}^{\alpha}}\right) \cong \frac{V\left(S_{i}^{\alpha}\right)}{M_{i}}=\frac{1}{M_{i}} \frac{\hat{p}_{i}\left(\hat{k}_{i} \hat{\beta}_{i}\right)^{2 \alpha}}{\Gamma\left(\hat{k}_{i}\right) \hat{k}_{i}^{2 \alpha}}\left(\Gamma\left(\hat{k}_{i}+2 \alpha\right)-\hat{p}_{i} \frac{\left(\Gamma\left(\hat{k}_{i}+\alpha\right)\right)^{2}}{\Gamma\left(\hat{k}_{i}\right)}\right)
$$

830

in the lognormal and gamma distributed case, respectively, and thus the variance in the case where $\sigma$ or $k$ is estimated for each sample separately. This estimate will be an

835 upper limit, as the gain in precision obtained by estimating a common CV is not accounted for.

The variance of $\hat{S(0)}$ and $\hat{S(T)}$ can be approximated by

$$
V\left(\hat{S}_{i}\right) \cong V\left(\hat{p}_{i}\right)\left(\bar{X}_{i}\right)^{2}+V\left(\bar{X}_{i}\right) \hat{p}_{i}^{2}+V\left(\bar{X}_{i}\right) V\left(\hat{p}_{i}\right)
$$


845 The approximations given above can only be used when the variance of $\bar{X}_{i}$ and $\hat{p}_{i}$ can be estimated. They can therefore not be applied to pooled stomach samples. In this case, the variance can be approximated by

850

$$
V\left(\hat{S_{i}^{\alpha}}\right) \cong \frac{V\left(S_{i}^{\alpha}\right)}{M_{i}}=\frac{1}{M_{i}}\left(e^{\alpha \hat{\sigma}^{2}(2 \alpha-1)}-\hat{p}_{i} e^{\alpha \hat{\sigma}^{2}(\alpha-1)}\right) \hat{p}_{i}{\overline{X_{i}}}^{2 \alpha}
$$

855 and

$$
V\left(\hat{S_{i}^{\alpha}}\right) \cong \frac{V\left(S_{i}^{\alpha}\right)}{M_{i}}=\frac{1}{M_{i}} \frac{1}{\Gamma(\hat{k}) \hat{k}^{2 \alpha}}\left(\Gamma(\hat{k}+2 \alpha)-\hat{p}_{i} \frac{(\Gamma(\hat{k}+\alpha))^{2}}{\Gamma(\hat{k})}\right) \hat{p}_{i} \bar{X}_{i}^{2 \alpha}
$$

860

in the lognormal and gamma distributed case, respectively.

The variance of $\hat{S(0)}$ and $\hat{S(T)}$ can be approximated by 


$$
V\left(\hat{S}_{i}\right) \cong \frac{V\left(S_{i}\right)}{M_{i}}=\frac{1}{M_{i}}\left(e^{\hat{\sigma}^{2}}-\hat{p}_{i}\right) \hat{p}_{i}{\overline{X_{i}}}^{2}
$$

and

875

$$
V\left(\hat{S}_{i}\right) \cong \frac{V\left(S_{i}\right)}{M_{i}}=\frac{1}{M_{i}}\left(\frac{\hat{k}+1}{\hat{k}}-\hat{p}_{i}\right) \hat{p}_{i}{\overline{X_{i}}}^{2}
$$

880 in the lognormal and gamma distributed case, respectively. The precision of these estimates depends entirely on the adequacy of the assumption of constant CV and the precision of the estimates of $\sigma$ and $k$. They are further lower limits, as the variance of $\hat{\sigma}$ and $\hat{k}$ are not taken into account. 
885 Appendix C

Estimation of bias of traditional methods for estimating the intake of individual prey types

The relative difference between the intake of prey $j$ calculated by the method presented here $\left(C_{3, j}\right)$ and by multiplying the weight percentage of $j$ in the total 890 stomach content, $\pi_{\text {tot }, j}$, to the total intake $\left(C_{4, j}\right)$ is

$$
\frac{C_{4, j}-C_{3, j}}{C_{3, j}}=\frac{\pi_{\text {tot }, j}}{\pi_{j}^{1-\alpha}} \frac{\rho_{\text {tot }}}{\left(\pi_{j} \rho_{j}+\left(1-\pi_{j}\right) \rho_{\text {res }}\right)} \frac{\sum_{i=1}^{N} E\left(S_{\text {tot }}^{\alpha}\right)}{\sum_{i=1}^{N} E\left(S_{j}^{\alpha}\right)}-1
$$

895

where

900

$$
\frac{\sum_{i=1}^{N} E\left(S_{t o t}^{\alpha}\right)}{\sum_{i=1}^{N} E\left(S_{j}^{\alpha}\right)}=\frac{\frac{\Gamma\left(k_{t o t}+\alpha\right)}{\Gamma\left(k_{t o t}\right) k_{t o t}^{\alpha}} \sum_{i=1}^{N} p_{t o t, i}\left(E\left(X_{t o t, i}\right)\right)^{\alpha}}{\frac{\Gamma\left(k_{j}+\alpha\right)}{\Gamma\left(k_{j}\right) k_{j}^{\alpha}} \sum_{i=1}^{N} p_{j, i}\left(E\left(X_{j, i}\right)\right)^{\alpha}}
$$


905 It is assumed that $\pi_{t o t, j}$ and $\pi_{j}$ remain constant over the period and no change occurs in the average stomach content over the sampling period. Note that in the special case $\alpha$ $=1$, the bias is entirely dependent on the difference in evacuation rates between prey.

If $\rho_{\text {tot }}=\rho_{j}=\rho_{\text {res }}$ and $k_{\text {tot }}=k_{j}$, equation (C1) can be reduced by inserting $E\left(X_{\text {tot }}\right)=\frac{p_{j}}{p_{\text {tot }}} \frac{E\left(X_{j}\right)}{\pi_{t o t, j}}$ :

$$
\frac{C_{4, j}-C_{3, j}}{C_{3, j}}=\left(\frac{\pi_{t o t, j}}{\pi_{j}} \frac{p_{t o t}}{p_{j}}\right)^{1-\alpha}-1
$$

Once again it is evident that in the case of $\alpha=1, C_{4}$ is only biased if evacuation rates differ between prey.

The bias introduced by disregarding the effect of other food in the stomach on evacuation (i.e. estimate food intake as $C_{5, j}$ ) is

$$
\frac{C_{5, j}-C_{3, j}}{C_{3, j}}=\frac{\frac{\rho_{j}}{N} \sum_{i=1}^{N} S_{i}^{\alpha}}{\frac{\left(\pi_{j} \rho_{j}+\left(1-\pi_{j}\right) \rho_{\text {res }}\right) \pi_{j}^{1-\alpha}}{N} \sum_{i=1}^{N} S_{i}^{\alpha}}-1=\frac{\rho_{j}}{\left(\pi_{j} \rho_{j}+\left(1-\pi_{j}\right) \rho_{\text {res }}\right) \pi_{j}^{1-\alpha}}-1
$$


925

It is again assumed that $\pi$ remains constant and no change in the average stomach content occurs over the sampling period. 


\section{Appendix D}

\section{$930 \quad$ Estimation of evacuation rates}

Andersen (2001) showed that the evacuation rate of individual prey types without exoskeleton is well described by a function of energy density of the prey. If several prey types occurred together, the evacuation rate of the total stomach content could be estimated from the average energy density of the total stomach content

935 (Andersen 2001). The relationship between evacuation rate and energy density was used here to calculate the evacuation rate of prey without exoskeleton. Energy densities were assumed to be equivalent to values given by Andersen (2001) and Pedersen and Hislop (2001). The energy density of benthic invertebrates was assumed to be $3.7 \mathrm{~kJ} \mathrm{~g}^{-1}$ based on the evacuation rate given by Rindorf (2004). The evacuation

940 rate of the total stomach content at each location was then calculated as a weighted average of the evacuation rate of non-exoskeleton prey (calculated from the average energy density of these prey) and the evacuation rate of exo-skeleton prey (taken to be equal to the value given for brown shrimp (Crangon crangon) in whiting of $27.5 \mathrm{~cm}$ by Andersen (2001)):

945

$$
\rho_{\text {tot }}=\left(0.0025 \pi_{\text {exo }}+0.152 E^{-0.86}\left(1-\pi_{\text {exo }}\right)\right) e^{0.078 t e m p}
$$


where $\pi_{e x o}$ is the proportion of the stomach content which consists of exoskeleton prey at each location, $1-\pi_{e x o}$ is the proportion of the stomach content which consists of prey without an exoskeleton, $E$ is average energy density of the prey without an 955 exoskeleton and temp is ambient temperature at the location. This is a modified version of the equation given by Andersen (2001) for whiting at $27.5 \mathrm{~cm}$. The size and temperature specific evacuation rates, $\rho_{L T}$, of the three prey types were: benthic invertebrates: 0.00042 (Rindorf 2004), crabs: 0.00021 (value given for brown shrimp in Andersen (1999)) and herring: 0.00037 (derived from the relationship given by 960 Andersen (2001) and an energy density of $4.3 \mathrm{~kJ}^{-1} \mathrm{~g}^{-1}$ (Pedersen and Hislop 2001)). With a predator length of $27.5 \mathrm{~cm}$, the evacuation rates were $0.050 \mathrm{e}^{0.078 t e m p}, 0.025$ $\mathrm{e}^{0.078 t e m p}$ and $0.032 \mathrm{e}^{0.078 t e m p}$ for benthic invertebrates, herring and crabs, respectively. 